\title{
Efecto en las características microestructurales y texturales de la impregnación de Ni y Sn sobre nanopartículas de $\mathrm{SnO}_{2}$
}

MATERIALS ENGINEERING

\section{Effect of the $\mathrm{Ni}$ and $\mathrm{Sn}$ impregnation in the microstructural and textural properties of $\mathrm{SnO}_{2}$ nanoparticles}

\author{
Carlos E. Ararat", Julián D. Urresta ${ }^{* *}$, Gerardo A. Torres ${ }^{* * *}$, \\ Jorge E. Rodríguez ${ }^{* * * *}$, Alfonso E. Ramírez ${ }^{\S^{*}}$ \\ * Grupo de Investigación en Catálisis, Departamento de Química, Universidad del Cauca, \\ Popayán, Colombia.cararat@unicauca.edu.co , \\ saramirez@unicauca.edu.co \\ ** Laboratorio de Investigación en Catálisis y Procesos LICAP, Departamento de Química, \\ Universidad del Valle, Cali, Colombia. \\ julian.urresta@correounivalle.edu.co \\ *** Unidad de Microscopía Electrónica, Universidad del Cauca, Popayán, Colombia. \\ gator@unicauca.edu.co \\ **** Grupo de Ciencia y Tecnología de Materiales Cerámicos CYTEMAC, Departamento de \\ Física, Universidad del Cauca, Popayán, Colombia. \\ jnpaez@unicauca.edu.co
}

(Recibido: Agosto 24 de 2010- Aceptado: Octubre 14 de 2011)

\begin{abstract}
Resumen
En este trabajo se estudió la síntesis y la caracterización de $\mathrm{SnO}_{2}$ y el efecto que tiene el Ni y $\mathrm{Sn}$, al ser incorporados a la estructura del $\mathrm{SnO}_{2}$ por impregnación. La obtención del $\mathrm{SnO}_{2}$ se realizó utilizando el método de precipitación controlada y el Sn y el Ni se adicionaron empleando el método de impregnación. Las técnicas TEM, EDX, XRD y área superficial se usaron para caracterizar los sólidos preparados. Se encontró que la impregnación con Ni conlleva a la formación de soluciones sólidas sustitucionales, dentro del rango de dopado empleado (hasta $3 \%$ en moles), y segregación sobre la superficie de las partículas de $\mathrm{SnO}_{2}$, lo que explica los efectos generados sobre las propiedades texturales del $\mathrm{SnO}_{2}$.
\end{abstract}

Palabras Clave: Óxido de estaño, Impregnación de Ni y Sn, Soluciones sólidas, Nano partículas.

\begin{abstract}
In this work, it was carried out the synthesis and characterization of $\mathrm{SnO}_{2}$ and determination of the microstructural and textural properties of $\mathrm{SnO}_{2}$ after $\mathrm{Ni}$ and $\mathrm{Sn}$ incorporation. The $\mathrm{SnO}_{2}$ nanoparticles were obtained by precipitation method and the incorporation of the $\mathrm{Sn}$ and Ni by impregnation method. The techniques TEM, EDX, XRD and surface area were used to characterize the solids. It was found that the impregnation of Ni leads to formation of substitutional solid solutions which should cause effects on the textural properties.
\end{abstract}

Keywords: Tin oxide, Ni and Sn impregnation, Solids solution, Nanoparticles 


\section{Introducción}

Los sistemas con base en óxidos se utilizan ampliamente en aplicaciones tecnológicas, particularmente el $\mathrm{SnO}_{2}$ se emplea en la elaboración de varistores, semiconductores electrodos transparentes, sensores de gas, paneles solares (Errico, 2007) y entre otras funcionalidades como catalizador heterogéneo en un amplio número de procesos industriales (Centi, 2008; Smith, 1998). Para esta última aplicación, la eficiencia en la reacción química en la cual pueden ser empleados, dependerá tanto de los precursores utilizados como de la forma como sean sintetizados (Perego \& Villa, 1997). Para la obtención de $\mathrm{SnO}_{2}$ se ha reportado el método del precursor polimérico Pechini con el cual se obtuvieron nanoestructuras bidimensionales (Mosquera \& Rodríguez, 2008 ), el método mecanoquímico con el cual se logran partículas con tamaño de cristalito entre 2-48 nm (Billiand Caplovicova, 2009), sol-gel (Kang et al. 2007), por envejecimiento hidrotérmico (He et al., 1999) para lograr un control más adecuado en el tamaño del cristal, suspensión coloidal para la construcción de electrodos de manera homogénea (Chappel \& Zaban, 2002) y combustión (Fraigi et al., 2001), entre otros.

Se ha reportado que la incorporación de un segundo elemento afecta las propiedades funcionales del $\mathrm{SnO}_{2}$, un ejemplo de ello es su capacidad oxidativa, la que se puede aumentar a nivel de actividad y de selectividad (Montenegro et al., 2007). Entre los elementos que frecuentemente se incorporan, para tal fin, se tienen el fósforo, antimonio, vanadio, cromo, molibdeno, manganeso y cobre (Meille, 2006). Estos sistemas se han utilizado para promover la oxidación del monóxido de carbono, dióxido de azufre e hidrocarburos saturados e insaturados, así como de otros compuestos orgánicos (Smith, 1998). Adicionando paladio a los sólidos, estos se pueden utilizar en la oxidación de metano (Sekizawa, et al., 2000) y en procesos de transesterificación de aceites y de dimetil oxalato, donde el óxido de estaño se mezcla con otros óxidos tales como: $\mathrm{SiO}_{2}, \mathrm{ZnO}$, y $\mathrm{TiO}_{2}$ (Urresta, et al., 2000; Wang, et al., 2004). Otro grupo de sólidos que contienen estaño, y que son de uso industrial, son los sistemas estaño-paladio
(Verdier, et al., 2003), estaño-rutenio (Toba, et al., 1999; Mendes, et al. 2001) estaño-platino, estañocobalto (Pouilloux, et al., 2000; Pouilloux, et al., 2001). Estos sistemas muestran una efectiva actividad en reacciones de deshidrogenación, deshidrociclación, craqueo, isomerización e hidrogenación de hidrocarburos y aceites (Kirk \& Othmer, 1962). Con relación al dopaje del $\mathrm{SnO}_{2}$ con níquel, se encuentran reportes de su incorporación por el método de "screen printing" para aumentar su capacidad como sensor de gases (Jain et. al., 2006), por medio del método del precursor polimérico para examinar sus propiedades magnéticas, caracteristicas estructurales y microscópicas (Aragón et al. 2010), por procesos cerámicos convencionales para estudiar su no-linealidad electrica (Wang et al., 2003), por sol-gel para aumentar la capacidad fotocatalítica del $\mathrm{TiO}_{2}(\mathrm{Khan} \& \mathrm{Kim}, 2009)$.

En este trabajo se sintetizó casiterita, $\mathrm{SnO}_{2}$ tipo rutilo, por el método de precipitación controlada y el material obtenido se utilizó como soporte de especies de Ni, Sn o Sn-Ni y se evaluó el efecto de la impregnación de estas especies sobre la estructura del $\mathrm{SnO}_{2}$.

\section{Parte experimental}

\subsection{Síntesis de $\mathrm{SnO}_{2}$}

La casiterita se obtuvo por el método de precipitación controlada (MPC) empleando $\mathrm{SnCl}_{2} .2 \mathrm{H}_{2} \mathrm{O}$ (98\%, Mallinckrodt) como precursor, siguiendo el proceso reportado previamente (Ararat, et al., 2007). El precursor de estaño se disolvió en agua desionizada, sometiendo el sistema a agitación magnética durante 15 minutos $\mathrm{y}$ en reposo durante $24 \mathrm{~h}$. Posteriormente se adicionó $\mathrm{NH}_{4} \mathrm{OH}$ (26-30\%, Mallinckrodt) a la solución, suministrado mediante un dosificador automático (Dosimat 685 Metrohm, Suiza) a una velocidad de $25 \mu \mathrm{L} / \mathrm{s}$, hasta que ella alcanzó un valor de $\mathrm{pH}$ de 6.25. El precipitado que se formó en el seno de la solución se filtró a gravedad y se vuelve a re-dispersar en una solución 0,05 M de dietilamina para eliminar los iones cloruro; este proceso se repitió cuatro veces hasta que la 
solución del lavado no genera un precipitado blanco al adicionar nitrato de plata amoniacal. El sólido húmedo, que se obtuvo al final de este proceso, se secó en una estufa a $70{ }^{\circ} \mathrm{C}$ durante 12 horas.

\subsection{Impregnación de Sn y Ni}

La introducción de Ni y Sn sobre el óxido de estaño se realizó por el método de impregnación. Para ello, se adicionó a $1.0 \mathrm{~g}$ del polvo cerámico sintetizado en suspensión con $10 \mathrm{~mL}$ de agua desionizada, los volumenes correspondientes de soluciones $0,1 \mathrm{M}$ de diferentes los precursores, $\mathrm{SnCl}_{2} \cdot 2 \mathrm{H}_{2} \mathrm{O}$ (98\%, Mallinckrodt) y $\mathrm{NiCl}_{2} \cdot 6 \mathrm{H}_{2} \mathrm{O}$ (98\%, Merck), según los porcentajes requeridos del metal. Luego, en agitación magnética constante y a una temperatura de $70^{\circ} \mathrm{C}$ hasta la eliminación del solvente. El sólido se secó a $100^{\circ} \mathrm{C}$ por 24 horas y después se calcinó a $400^{\circ} \mathrm{C}$ en un horno circular RACK SICO TEMP MOD, bajo un flujo de aire de $10 \mathrm{~L} / \mathrm{h}$ y una rampa de calentamiento de $4^{\circ} \mathrm{C} / \mathrm{min}$, partiendo de $25^{\circ} \mathrm{C}$ y llevando la muestra a $110^{\circ} \mathrm{C}(1$ hora $)$ y a $450^{\circ} \mathrm{C}(8$ horas). En la Tabla 1 se indican los sistemas preparados.

Tabla 1. Sólidos preparados y sus respectivos porcentajes de Niy $\mathrm{Sn}$

\begin{tabular}{ccc}
\hline Sólido & $\mathbf{N i , \% p / p}$ & Sn, \% p/p \\
\hline $\mathrm{SnO}_{2}$ & 2 & \\
$\mathrm{Ni} 2 \% / \mathrm{SnO}_{2}$ & 3 & \\
$\mathrm{Ni} \% / \mathrm{SnO}_{2}$ & & 2 \\
$\mathrm{Sn} 2 \% / \mathrm{SnO}_{2}$ & & 3 \\
$\mathrm{Sn} 3 \% / \mathrm{SnO}_{2}$ & \\
$\mathrm{Sn} 2 \%-\mathrm{Ni} 0.5 \% / \mathrm{SnO}_{2}$ & 0.5 & 2 \\
\hline
\end{tabular}

\subsection{Caracterización de los sólidos}

Para determinar las fases cristalinas presentes en los sólidos sintetizados se utilizó Difracción de Rayos X (XRD), utilizando para ello el Difractómetro RIGAKU 2200 con fuente de $\mathrm{Cu}$ $(\mathrm{CuK} \alpha=1.54056 \AA)$ a un paso de 0.02 grados y un tiempo fijo de 0.3 segundos, barriendo el rango 20 y $80^{\circ}$. Para calcular los parámetros de red, a partir de los datos obtenidos de la difracción, se utilizó el software libre Full-Prof. Para determinar el área superficial de los sólidos se usó un Micromeritics ASAP 2010; el parámetro se calculó por el método BET a partir de las isotermas de adsorción desorción de $\mathrm{N}_{2}$ a 77K. El tamaño y la morfología de las partículas impregnadas se determinaron utilizando un Microscopio Electrónico de Barrido (SEM), marca TOPCON Sm-300, y un Microscopio Electrónico de Transmisión (TEM) Jeol JEM 1200EX; para el análisis de dispersión de rayos X (EDX) se utilizó la microsonda EDX PGT, PRISM-digital spectrometer.

\section{Resultados y discusión}

\subsection{Caracterización de los sólidos}

Los resultados del análisis por difracción de rayos $\mathrm{X}$ de los sistemas obtenidos mostraron que la principal fase cristalina corresponde al $\mathrm{SnO}_{2}$ (casiterita PDF 41-1445), con una estructura tetragonal tipo rutilo (figura 1). Además, debido a la poca cantidad de los dopantes, Ni y Sn, se puede observar que su detección por XRD es imperceptible con relación al soporte, figura 1(a). Sin embargo, se observa un leve desplazamiento de los picos de difracción del $\mathrm{SnO}_{2}$ al impregnar el soporte con níquel, más evidente en el pico (100) (figura 1(b)), comportamiento que se realza al aumentar el porcentaje másico del dopante.

El evidente desplazamiento de los picos de difracción, figura $1(\mathrm{~b})$, indica que el $\mathrm{Ni}^{2+}$ se podría introducir en la estructura del $\mathrm{SnO}_{2}$, sustituyendo al $\mathrm{Sn}^{+4}$ y generando vacancias de oxígeno para restablecer el balance de carga local, dando origen a soluciones sólidas sustitucionales. Esto se puede representar a través de la reacción indicada en la Ec. (1):

$$
\mathrm{NiO} \text { (ads) } \stackrel{\mathrm{SnO}_{2}}{\longrightarrow} \mathrm{NiSn}_{(\text {estructural })}+\mathrm{Vö}+1 / 2 \mathrm{O}_{2}
$$

Al realizar el cálculo de los parámetros de red de algunos de los sistemas (Tabla 2) se puede observar que el volumen de la celda, para los sistemas que contienen níquel, decrece ligeramente en comparación con el volumen de 


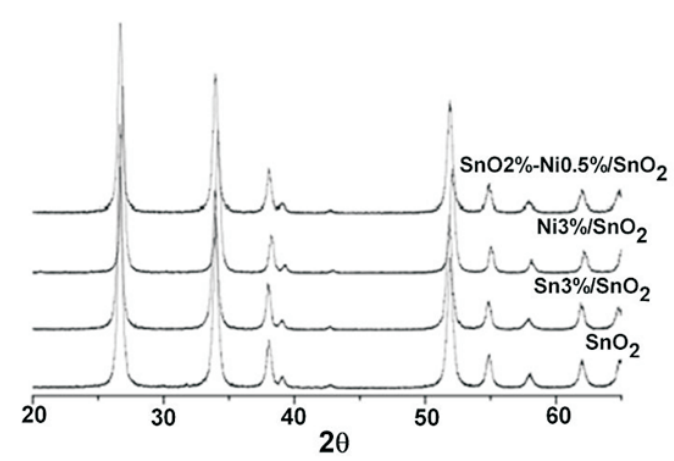

b

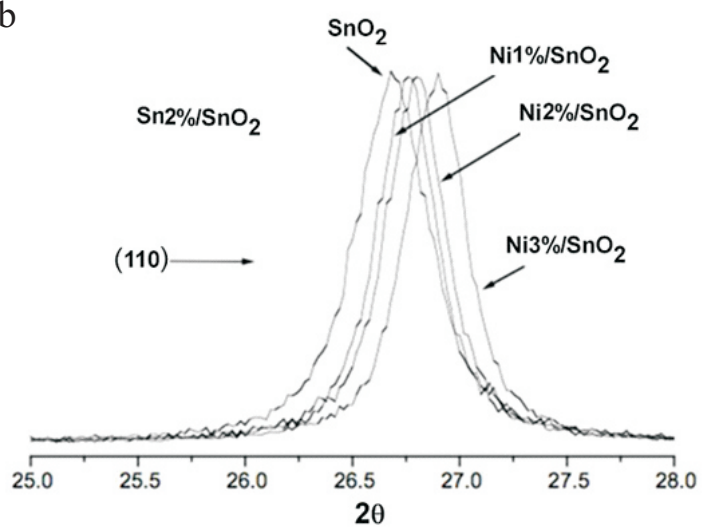

Figura 1. Difractógramas correspondientes a los sistemas de interés (a) y los perfiles del pico (100) para diferentes concentraciones de $\mathrm{Ni}(b)$

Tabla 2. Parámetros de red calculados para los sistemas analizados

\begin{tabular}{ccccc}
\hline Sistema & \multicolumn{4}{c}{ Parámetros de red } \\
\cline { 2 - 5 } & \multicolumn{1}{c}{$\mathrm{a}$} & $\mathrm{c}$ & $\mathrm{a} / \mathrm{c}$ & Vcelda \\
& $(\AA)$ & $(\AA)$ & $(\mathrm{A} 3)$ \\
\hline $\mathrm{SnO} 2$ & 4.7213 & 3.1801 & 1.4846 & 70.888 \\
$\mathrm{Sn} 3 \% / \mathrm{SnO}_{2}$ & 4.7040 & 3.1749 & 1.4816 & 70.253 \\
$\mathrm{Ni3} \% / \mathrm{SnO}_{2}$ & 4.6834 & 3.1655 & 1.4795 & 69.432 \\
$\mathrm{Sn} 2 \% \mathrm{Ni} 0.5 \% / \mathrm{Sn}_{2} \mathrm{O}$ & 4.7144 & 3.1744 & 1.4830 & 70.551 \\
\hline
\end{tabular}

celda calculado para el $\mathrm{SnO}_{2}$; para los sistemas impregnados con $\mathrm{Sn}$, sus parámetros de red no sufren modificaciones apreciables.

Estos resultados son de gran interés debido a la controversia que existe en la literatura especializada con relación a lo que ocurre cuando partículas de $\mathrm{SnO}_{2}$ son dopadas con $\mathrm{Ni}^{2+}$. Aragón et. al., 2010, doparon $\mathrm{SnO}_{2}$ con $\mathrm{Ni}$ (hasta un $10 \%$ en moles) para estudiar su comportamiento magnético. Concluyendo que solo se producían soluciones sólidas para contenidos de níquel por debajo $\sim 1 \%$ en moles, sin desplazamiento apreciable de los picos de difracción en los difractogramas correspondientes a estas muestras. Aragón et. al., 2010 justificaron la conformación de soluciones sólidas, para los contenidos de $\mathrm{Ni}^{2+}$ indicados, por el incremento que observaron del ancho de los picos, a la altura media del mismo (FWHM por sus siglas en inglés), con el aumento del contenido de níquel. Para mayores contenidos de níquel, proponen que el ión se segregaría en la superficie de las partículas, ocupando sitios intersticiales. Utilizando espectroscopia Raman, verificaron la ocurrencia de estas soluciones sólidas para concentraciones de $\mathrm{Ni}^{2+}$ menores al $1 \%$.

Por otro lado, Jain et. al. (2006), estudiaron el dopado de películas gruesas de $\mathrm{SnO}_{2}$ con níquel, para sensores de gases, y observaron que la componente en menor concentración, el $\mathrm{Ni}^{2+}$, se segregaba sobre la superficie del $\mathrm{SnO}_{2}$, aún para muestras con $30 \%$ en moles de níquel. 
Considerando los resultados de este trabajo, el desplazamiento de los picos de difracción, figura 1(b), evidencia la formación de soluciones sólidas pero no excluye que, además, se segregue parte de los iones $\mathrm{Ni}^{2+}$ sobre la superficie de las partículas de $\mathrm{SnO}_{2}$.

Además, la presencia de estos dopantes puede ocasionar alteraciones en las propiedades texturales del $\mathrm{SnO}_{2}$. Según las isotermas de adsorción - desorción de $\mathrm{N}_{2}$, figura 2, los sistemas presentaron curvas características tipo $V$, según la clasificación IUPAC, indicando que la interacción con el adsorbente es débil.

Por otro lado, la aparición de histéresis a $P / P$ o 0,8 indica la presencia de poros mayores que los microporos. Los sistemas con $\mathrm{Ni}, \mathrm{Sn}$ y Ni-Sn, estudiados en este trabajo, presentaron en estas

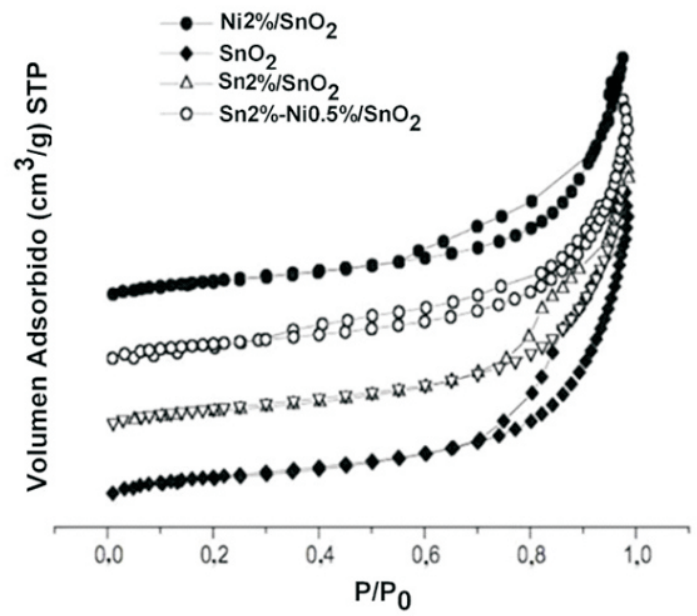

Figura 2. Isotermas de adsorción desorción de $\mathrm{N}_{2}$ a $77 \mathrm{~K}$ para los sistemas estudiados curvas una histéresis tipo $\mathrm{H}_{3}$ lo que indica que a sólido presenta poros de placa paralelas "slitshaped" (Rouquerol et al., 1999).

Para el $\mathrm{SnO}_{2}$ su distribución de volumen de poro fue cercano a los $0.067 \mathrm{~cm}^{3} / \mathrm{g}$ presentando un diámetro de $139 \AA$ A. Igualmente, se puede observar que la impregnación del $\mathrm{SnO}_{2}$ con $\mathrm{Ni}$ o $\mathrm{Sn}$, aunque no ocasiona grandes cambios en su área superficial, produce variaciones en el volumen y el diámetro de poro. La Tabla 3 resume las principales características texturales de los sólidos.

Los cambios en la porosidad en estos sistemas, al impregnar con Ni o Sn, son confirmados por la Figura 3 donde se observa disminución del volumen de poro y ensanchamiento en la distribución del tamaño de poro.

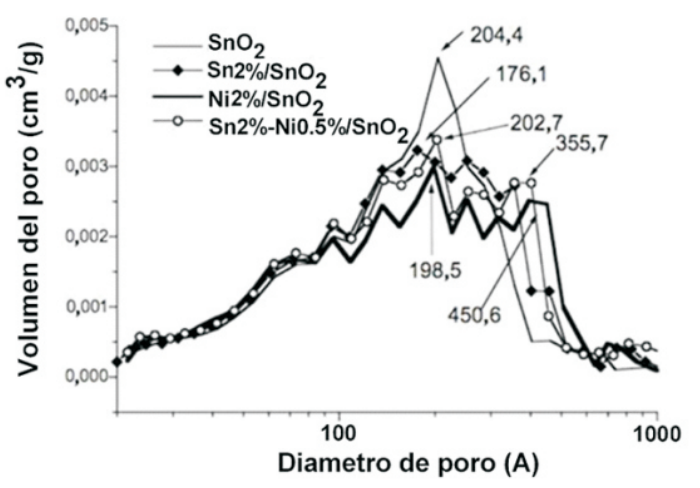

Figura 3. Distribución de tamaño de poro para algunos de los sistemas estudiados

Tabla 3. Propiedades texturales de los sistemas

\begin{tabular}{|c|c|c|c|}
\hline Sistema & $\begin{array}{c}\text { Area } \mathrm{S}_{\mathrm{BET}}, \\
\mathrm{m}^{2} / \mathrm{g}\end{array}$ & $\begin{array}{c}\text { V de poro BJH, } \\
\mathrm{cm}^{3} / \mathrm{g}\end{array}$ & $\begin{array}{c}\text { Diámetro de poro, } \\
(\AA ̊)\end{array}$ \\
\hline $\mathrm{SnO}_{2}$ & 20.1 & 0.066361 & 139.19 \\
\hline $\mathrm{Sn} 2 \% / \mathrm{SnO}_{2}$ & 20.2 & 0.067168 & 141.92 \\
\hline $\mathrm{Ni} 2 \% / \mathrm{SnO}_{2}$ & 18.9 & 0.055388 & 119.09 \\
\hline $\mathrm{Sn} 2 \% \mathrm{Ni} 0.5 \% / \mathrm{Sn}_{2} \mathrm{O}$ & 19.5 & 0.060626 & 129.73 \\
\hline
\end{tabular}


Se destaca la diferencia que presentaron los sistemas $\mathrm{Sn} 2 \% / \mathrm{SnO}_{2}$ y Sn $2 \% \mathrm{Ni} 0.5 \% / \mathrm{SnO}_{2}$, lo que pone en evidencia el efecto que tiene la presencia del níquel en el sistema sobre la distribución del tamaño de poro, a pesar de estar en poca cantidad.

Las micrografías obtenidas de los sistemas de interés, Figura 4, muestran aglomeración de las partículas primarias. Para los sistemas impregnados con estaño, Figura 4(a), es evidente una mayor aglomeración, con respecto al material de partida $\left(\mathrm{SnO}_{2}\right)$, así como una forma irregular de las partículas. Algo similar ocurre con los sistemas que contienen níquel, Figura 4(b). Para el sistema $\mathrm{Sn} 2 \%-\mathrm{Ni} 0.5 \% / \mathrm{SnO}_{2}$, Figura 4(c), se observa un grado menor de aglomeración y una distribución de tamaño de partícula más heterogéneo.
El análisis realizado con la microsonda (EDX), Figura 5, muestra la presencia de $\mathrm{Ni}$ en los materiales ubicado, preferencialmente, en la superficie de los aglomerados, resultado que verificaría la segregación de $\mathrm{Ni}^{2+}$ en estos lugares además de su incorporación en la red del $\mathrm{SnO}_{2}$, para sustituir el $\mathrm{Sn}^{4+}$, y conformar las soluciones sólidas que se evidenciaron con los resultados de DRX (Figura 1(b)).

Con base en los resultados obtenidos, a continuación se propone un modelo para justificar la formación de una gran cantidad de aglomerados en el sistema impregnado con Sn (Figura 6).

b
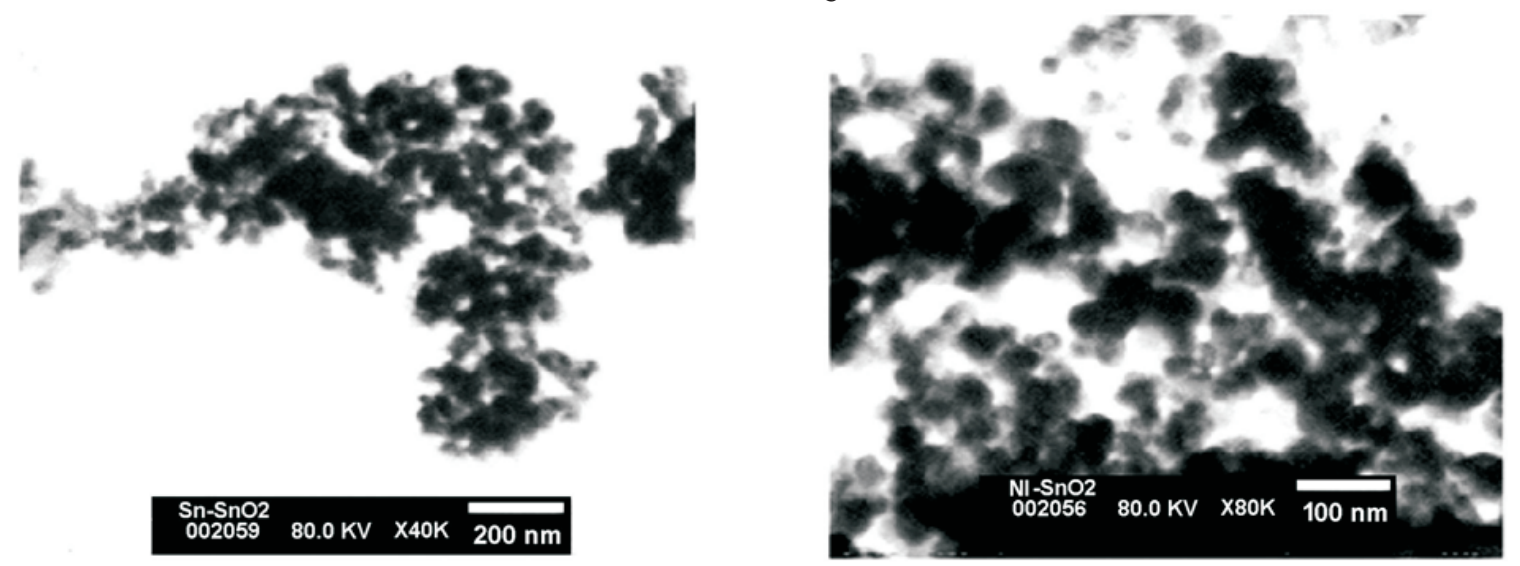

c

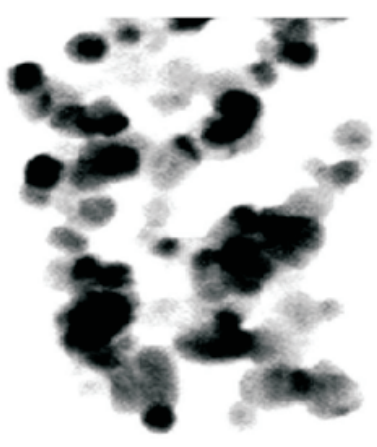

n-N1/SHO2 $80.0 \mathrm{KV} \times 200 \mathrm{~K} \quad 20 \mathrm{~nm}$

Figura 4. Fotomicrografias obtenidas por Microscopía Electrónica de Transmisión correspondientes a los sistemas. a) Sn $2 \% / \mathrm{SnO}_{2}$, b) $\mathrm{Ni} 2 \% / \mathrm{SnO}_{2}$, c) $\mathrm{Sn} 2 \%-\mathrm{NiO}, 5 \% / \mathrm{SnO}_{2}$ 


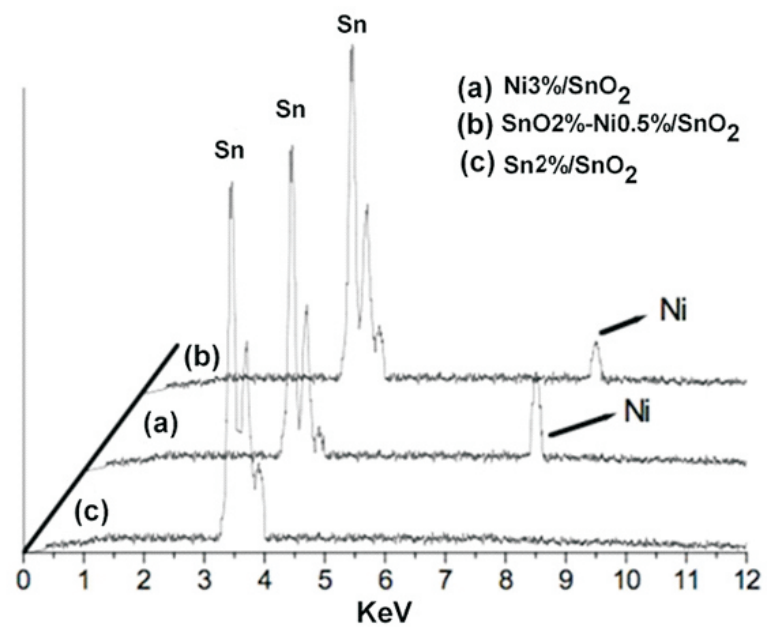

Figura 5. Análisis con microsonda (EDX) a los sistemas con base en estaño.

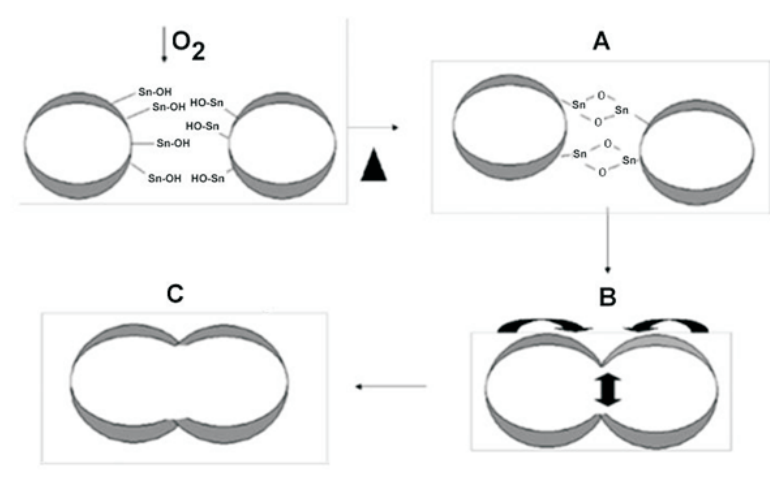

Figura 6. Modelo propuesto para justificar la formación de cuellos entre las partículas de los sistemas estudiados

La presencia del Sn superficial favorecería una fuerte interacción entre las partículas durante el proceso de calcinación del sistema ya que la presencia de grupos funcionales $\mathrm{Sn}-\mathrm{OH}$, en la superficie de las mismas, y la del $\mathrm{O}_{2}$ del aire favorecerían la formación de enlaces del tipo SnO-Sn, Figura 6A. Por otro lado, dada la alta difusión superficial, a bajas temperaturas, que se presenta en el $\mathrm{SnO}_{2}$ favorecería la formación de cuellos entre las partículas, Figura 6B, y por lo tanto la formación de agregados, Figura 6C, como los que se observan en la micrografía de la figura 4(a), que afectan las características texturales de los sólidos sintetizados (ver Tabla 3 ).
Por otro lado, para el caso del material impregnado $\mathrm{Ni}-\mathrm{SnO}_{2}$, la presencia del $\mathrm{Ni}$ en el sistema, y la sustitución del $\mathrm{Sn}^{+4}$ por el Ni ${ }^{+2}$ tal como se indicó en la ecuación (1), se generan vacancias de oxígeno en la estructura para compensar el desbalance de carga generada por la diferencia del estado de oxidación de los cationes involucrados, lo que ocasiona un aumento en el transporte de masa, a nivel superficial, y por lo tanto en el grado de coalescencia de las partículas (aumento en el tamaño de los cuellos entre partículas) tal como se indica en la figura $6 \mathrm{C}$ y que se evidencia en la figura 4(b).

\section{Conclusiones}

En este trabajo se sintetizaron, por rutas químicas, $\mathrm{SnO}_{2}, \mathrm{SnO}_{2}-\mathrm{Sn}$ y $\mathrm{SnO}_{2}-\mathrm{Ni}$. La adición del $\mathrm{Ni}^{2+}$ generó, principalmente, soluciones sólidas con el $\mathrm{SnO}_{2}$, debido a la sustitución del $\mathrm{Sn}^{+4}$ por el $\mathrm{Ni}^{+2}, \mathrm{y}$ otro porcentaje del ión se segrego sobre la superficie de los aglomerados. La incorporación del $\mathrm{Sn}$ y el Ni, a las nanopartículas de $\mathrm{SnO}_{2}$, ocasionó efectos en las propiedades texturales del $\mathrm{SnO}_{2}$, lo que afectaría la funcionalidad superficial de estos sólidos, así como ocurre con su comportamiento magnético (Aragón et. al., 2010) y el sensado de gases (Jain et. al., 2006).

Específicamente, la adición de $\mathrm{Sn}$ o $\mathrm{Ni}$ al $\mathrm{SnO}_{2}$, utilizando el método de impregnación, favoreció la conformación agregados que afectan la porosidad y el área superficial de los sistemas conformados. Hay que estudiar más cuidadosamente el sistema que contiene tanto $\mathrm{Sn}$ como Ni, Sn-Ni/SnO 2 , para justificar por qué en él no se favorece la agregación como sí sucede en los sistemas individuales, $\mathrm{Sn}-\mathrm{SnO}_{2}$ y Ni-SnO${ }_{2}$.

\section{Agradecimientos}

Agradecimientos a COLCIENCIAS proyecto 1103-14-17900 y a la Vicerrectora de Investigaciones (VRI) de la Universidad del Cauca por la financiación económica de este proyecto. Agradecimiento especial al proyecto PROALERTA CyTED por la financiación de la pasantía del Sr. Carlos Ararat al LIEC AraraquaraBrasil. 


\section{Referencias bibliográficas}

Aragón, F.H., Coaquira, J.A.H., Hidalgo, P., Brito, S.L.M., Gouvêa, D., \& Castro, R.H.R. (2010). Experimental study of the structural, microscopy and magnetic properties of Ni-doped $\mathrm{SnO}_{2}$ nanoparticles. Journal of Non-Crystalline Solids 356,29602964

Ararat, C.E., Mosquera, A.A., Castro, M.S., Parra, R., \& Rodríguez-Páez, J.E. (2007). Synthesis of $\mathrm{SnO}_{2}$ nanoparticles through the Controlled Precipitation Route. Materials Chemistry and Physics 101, 433-440.

Billiand, P., \& Caplovicova, M. (2009). Synthesis of nanocrystalline $\mathrm{SnO}_{2}$ powder from $\mathrm{SnCl}_{4}$ by mechanochemical processing. Powder Technology 191 (3), 235-239.

Centi, G., \& Perathoner, S. (2008). Catalysis, a driver for sustainability and societal challenges. Catalysis Today 138, 6976.

Chappel, S., \& Zaban, A. (2002). Nanoporous $\mathrm{SnO}_{2}$ electrodes for dye-sensitized solar cells: Improved cell performance by the synthesis of 18 $\mathrm{nm} \mathrm{SnO}$. Solar Energy Materials and Solar Cells 71 (2) 141-152.

Errico, L.A., (2007). Study of the semiconductors $\mathrm{SnO}$ and $\mathrm{SnO}_{2}$. Condensed Matter. 389, (1), 140144.

Fraigi, L.B., Lamas, D.G., \& Walsoe de Reca, N.E. (2001). Comparison between two combustion routes for the synthesis of nanocrystalline $\mathrm{SnO}_{2}$ powders. Materials Letters 47 (4-5), 262-266.

He Y., Li, Y., Yu, J., \& Qian, Y. (1999). Chemical control synthesis of nanocrystalline $\mathrm{SnO}_{2}$ by hydrothermal reaction. Materials Letters 40 (1), 23-26.

Kang, S.-Z., Yang, Y., \& Mu, J. (2007) Solvothermal synthesis of $\mathrm{SnO}_{2}$ nanoparticles by oxidations of $\mathrm{Sn}^{+2}$ ions at the water-oil interface. Colloids and Surface A: Physicochemical and Engineering Aspects 298 (3), 280-283.
Khan, R., \& Kim, T-J. (2009). Preparation and application of visible-light-responsive Ni-doped and $\mathrm{SnO}_{2}$-coupled $\mathrm{TiO}_{2}$ nanocomposite photocatalysts Journal of Hazardous Materials $163(2-3), 11791184$

Kirk, R., \& Othmer, D. (1962). Enciclopedia de Tecnología Química. México: Unión Tipográfica Editorial Hispano América.

Jain, K., Pant, R.P., \& Lakshmikumar, S.T. (2006). Effect of Ni doping on thick film $\mathrm{SnO}_{2}$ gas sensor. Sensors and Actuators B 113, 823829

Meille, V. (2006). Review on methods to deposit catalysts on structured surfaces. Applied Catalysis A: General, 315, 1-17.

Mendes, M., Santos, O.A., \& Silva, A.M. (2001). Hydrogenation of oleic acid over ruthenium catalysts. Applied Catalysis A: General 217, 253262.

Montenegro, A., Ponce, M., Castro, M.S., \& Rodríguez-Páez, J.E. (2007). $\mathrm{SnO}_{2}-\mathrm{BiO}_{3}$ and $\mathrm{SnO}_{2}-\mathrm{SbO}_{3}$ gas sensors obtained by soft chemical method. Journal European of Ceramic Society 27, 4143-4146.

Mosquera, A., \& Rodríguez, J.E. (2008). Obtención de nanoestructuras bidimensionales de $\mathrm{SnO}_{2}$ utilizando el método de pechini: Estudio de la conformación de la resina. Boletín de la Sociedad Española de Cerámica y Vidrio 47 (5), 278-286.

Perego, C., \& Villa, P. (1997). Catalyst preparation methods. Catalysis Today 34, 281-305.

Pouilloux, Y., Autin, F., \& Barrault, J. (2000). Selective hydrogenation of methyl oleate into unsaturated alcohols relationships between catalytic proprieties and composition of cobalt-tin catalysts. Catalysis Today 63, 87-100.

Pouilloux, Y., Barrault, J., \& De Oliveira, K. (2001). Selective Hydrogenation of methyl oleate into unsaturated alcohols in the presence of cobalt-tin catalysts over zinc oxide catalysts. Journal of Catalysis 204, 230-237. 
Rouquerol, F., Rouquerol, J., \& Sing, K. (1999). Adsorption by powders and porous solids: principles, methodology and applications. Academic Press.

Sekizawa, K., Widjaja, H., Maeda, S., Ozawa, Y., \& Eguchi, K. (2000). Low Temperature of methane over $\mathrm{Pd} / \mathrm{SnO}_{2}$ catalyst. Applied Catalysis A: General 200, 211-217.

Smith, P.J. (1998). Chemistry of Tin. 2a. Ed. Great Britain: Blackie Academic \& Professional Chapman \& Hall.

Toba, M., Tanaka, Sh., Niwa, I., Mizukami, F., Koppány, Z., Guczi, L., Cheah, K-Y. , \& Tang. ThS. (1999). Synthesis of alcohols and diols by hydrogenation of carboxylic acids and esters over $\mathrm{Ru}-\mathrm{Sn}-\mathrm{Al}_{2} \mathrm{O}_{3}$ catalysts. Applied Catalysis A: General 189, 243-250.

Urresta, J., Ramírez, A., Martínez, C., \& Vargas, L. (2000). Transesterificación del aceite de palma utilizando catalizadores soportados a base de $\mathrm{Sn}$ y Ni. Palmas 21,376.

Verdier, S., Didillon, B., Morin, S., \& Jumas, J.C. (2003). Pd-Sn $/ \mathrm{Al}_{2} \mathrm{O}_{3}$ Catalyst from colloidal oxide syntesis. Journal of Catalysis, 218, 280-287.

Wang, J. F., Chen, H. C., Wang, W. X., Su, W. B., \& Zang, G. Z. (2003) Electrical nonlinearity of (Ni, Ta) doped $\mathrm{SnO}_{2}$ varistors. Materials Science and Engineering B 99, 465-469.

Wang, S., Ma, X., \& Yang, X. (2004). Transesterification of Dimethyl Oxalate with Phenol under $\mathrm{SnO}_{2} / \mathrm{SiO}_{2}$ Catalysts. Industrial Engineering Chemical Research, 4027-4030. 